\title{
La Ligue mondiale pour la réforme sexuelle : La science au service de l'émancipation sexuelle?
}

\section{Florence Tamagne}

\section{(2) OpenEdition}

1 Journals

\section{Édition électronique}

URL : https://journals.openedition.org/clio/1751

DOI : 10.4000/clio. 1751

ISSN : 1777-5299

Éditeur

Belin

\section{Édition imprimée}

Date de publication : 1 novembre 2005

Pagination : 101-121

ISBN : 2-85816-821-0

ISSN : $1252-7017$

Référence électronique

Florence Tamagne, «La Ligue mondiale pour la réforme sexuelle : La science au service de l'émancipation sexuelle? », Clio. Histoire, femmes et sociétés [En ligne], 22 | 2005, mis en ligne le 01 décembre 2007, consulté le 22 avril 2022. URL : http://journals.openedition.org/clio/1751 ; DOI : https://doi.org/10.4000/clio.1751

Ce document a été généré automatiquement le 22 avril 2022.

Tous droits réservés 


\title{
La Ligue mondiale pour la réforme sexuelle : La science au service de l'émancipation sexuelle?
}

\author{
Florence Tamagne
}

\begin{abstract}
« Ce qui à cette époque était regardé comme excentrique et Utopique en est maintenant venu à être accepté comme une évidence dans presque tous les pays civilisés. ${ }^{1}$
\end{abstract}

1 Les fondements de la sexologie furent posés, à partir de la fin du XIXe siècle, à travers les œuvres de Krafft-Ebing et Freud en Autriche, d'Iwan Bloch, Magnus Hirschfeld et Albert Moll en Allemagne, d'Havelock Ellis en Angleterre et d'Auguste Forel en France. Cette nouvelle "science de la sexualité", préoccupée par les troubles sexuels, éprise d'hygiénisme, mais aussi séduite par le projet eugéniste, se voulait porteuse d'utilité sociale. De nombreux médecins s'engagèrent alors activement dans le débat sur la sexualité, croisant la route des mouvements de réforme sexuelle, qu'ils soient d'inspiration néo-malthusienne, féministe, et/ou libertaire. Ces différents courants allaient trouver, à partir de 1928, la possibilité de conjuguer leurs efforts au sein d'une même organisation, la Ligue mondiale pour la réforme sexuelle sur une base scientifique (L.M.R.S.). Fondée par Magnus Hirschfeld, célèbre pour sa lutte en faveur de la reconnaissance des homosexuels en Allemagne, elle s'inscrivait logiquement dans le prolongement de ses actions antérieures ${ }^{2}$ : faire progresser les droits des minorités sexuelles par la réflexion scientifique, légitimer les revendications homosexuelles en les intégrant dans le mouvement pour la réforme sexuelle.

2 La Ligue prenait naissance dans une période de "crise sexuelle ${ }^{3}$ marquée par les affrontements, plus ou moins violents selon les pays, autour du birth control et de l'examen prénuptial, du divorce et de la prostitution, de l'homosexualité ou du dépistage des "dégénérescences ». Ce climat de tension transparaissait dans le vœu émis par la Ligue d'atteindre les objectifs fixés « en faisant appel à l'intellect, et non aux émotions» et de «soigneusement s'abstenir d'attaques personnelles contre les 
opposants $»^{4}$. Pourtant, la L.M.R.S. allait être déchirée entre des tendances contradictoires, alors même que la montée des périls, la crise économique et sociale des années 30 et l'arrivée des nazis au pouvoir en Allemagne rendaient le combat pour la réforme sexuelle de plus en plus difficile à mener dans une perspective qui se voulait à la fois scientifique et apolitique.

\section{La Ligue mondiale pour la réforme sexuelle, une utopie scientifique?}

3 La Ligue mondiale pour la réforme sexuelle fut officiellement fondée lors du Congrès de Copenhague $e^{5}$ qui se tint $\mathrm{du} 1^{\mathrm{er}}$ au 5 juillet 1928. C'est durant ce Congrès, historiquement le deuxième de la Ligue, après celui de Berlin de $1921^{6}$, que furent présentés les objectifs, le programme, la constitution et les principes fondamentaux de la Ligue ainsi que les grandes lignes de son organisation, même si ces textes connurent par la suite des aménagements parfois importants ${ }^{7}$. Le pouvoir décisionnel revenait au Président de la Ligue, aidé d'un Comité Central exécutif de 5 membres, qui devaient théoriquement se réunir tous les trimestres, ainsi qu'à un Comité International composé de représentants (trois au maximum) des pays membres, élu lors de l'Assemblée Générale qui se tenait à l'occasion du Congrès International. Le siège habituel de la L.M.R.S. était situé à Berlin, dans l'Institut pour la Science Sexuelle (Institut für Sexualwissenschaft) de Magnus Hirschfeld, ce qui marquait bien la prépondérance, à ses origines, de la sexologie allemande.

4 Une résolution générale résumait les ambitions de la Ligue, et identifiait les cibles privilégiées de son action : «Le Congrès International pour la Réforme Sexuelle sur une Base Scientifique en appelle à toutes les législatures, à la presse et aux peuples de tous les pays pour aider à créer une nouvelle attitude sociale et légale (basée sur la connaissance qui a été acquise grâce à la recherche scientifique en matière de biologie sexuelle, de psychologie et de sociologie) à l'égard de la vie sexuelle des hommes et des femmes $\|^{8}$. Le programme de la Ligue reprenait celui élaboré par Magnus Hirschfeld pour le Congrès de Berlin : «(1) l'égalité politique, économique et sexuelle des hommes et des femmes ; (2) la libération du mariage (et spécialement le divorce) de la tyrannie de l'Eglise et de l'Etat; (3) le contrôle de la conception, de manière à ce que la procréation ne soit engagée que de manière volontaire et responsable; (4) l'amélioration de la race par l'application des connaissances eugéniques; (5) la protection de la mère célibataire et de l'enfant illégitime ; (6) une attitude rationnelle à l'égard des personnes sexuellement anormales, et spécialement à l'égard des homosexuels, hommes et femmes ; (7) la prévention de la prostitution et des maladies vénériennes; (8) les perturbations de l'instinct sexuel doivent être regardées comme des phénomènes plus ou moins pathologiques, et non, comme par le passé, comme des crimes, des vices ou des péchés; (9) ne doivent être considérés comme criminels que les actes sexuels qui portent atteinte aux droits sexuels d'une autre personne. Les actes sexuels entre adultes responsables, conclus d'un commun accord, doivent être regardés comme ne relevant que de la vie privée de ces adultes; (10) une éducation sexuelle systématique ». Ces points ne différaient pas sur l'essentiel de ceux mis en avant par les autres mouvements de réforme sexuelle, qu'ils soient progressistes ou révolutionnaires ${ }^{9}$, si ce n'est par l'attention particulière portée à la question 
homosexuelle ainsi que par la volonté d'envisager la question sexuelle de manière globale, et non pas branche par branche.

5 Forte de la notoriété de ses membres - Auguste Forel, Havelock Ellis et Magnus Hirschfeld étaient présidents d'honneur - et de sa vocation internationale, la Ligue entendait faire pression sur les gouvernements afin d'obtenir des réformes, notamment en matière juridique et pénale, et contribuer à l'information de l'opinion publique par la publication, ou le soutien à la publication de travaux, qu'ils soient techniques ou de vulgarisation, à condition qu'ils encouragent la «réforme sexuelle sur une base scientifique », la production d'un Journal International de la Réforme Sexuelle ${ }^{10}$, la tenue de congrès internationaux, la propagande par le biais de conférences, la fourniture d'informations aux législatures de tous les pays et une assistance en matière de législation sexuelle. Ces méthodes rappelaient celles employées par le WhK, qui avait fait de l'édition scientifique et du lobbying parlementaire les axes clés de son action, si ce n'est que la Ligue n'eut pas recours à l'arme de la pétition. Dans une motion lue lors du Congrès de Copenhague par Stella Croissant au nom de la «Ligue de la Régénération humaine ", Eugène Humbert et Georges Hardy affirmaient ainsi être "fermement convaincus que l'autorité des membres du Congrès sera assez puissante pour contribuer à faire que la question sexuelle ne soit plus la question 'tabou' par excellence, mais soit soumise, sous tous ses aspects, à la discussion scientifique et publique $»^{11}$.

6 La capacité d'attraction de la Ligue fut indéniable. Si l'on comptait 70 délégués internationaux à Copenhague en 1928, ils étaient 350 à Londres en 1929, le Portugal étant le seul pays européen non représenté, et 2000 à Vienne en 1930. En 1932, la Ligue rassemblait 190000 membres, la plupart répartis dans les diverses associations affiliées $^{12}$. La Ligue se voulait avant tout le catalyseur d'actions nombreuses mais en partie dispersées. Le Bund für Mutterschutz en Allemagne, la British Society for the Study of Sex Psychology (BSSSP) en Angleterre adhérèrent par exemple à la Ligue et des branches nationales furent créées avec des succès variés. L'adhésion était aussi possible à titre individuel ${ }^{13}$. On distingue, parmi les personnalités de premier plan, Norman Haire et Dora Russell pour l'Angleterre, Margaret Sanger pour les États-Unis, Max Hodann et Helene Stöcker pour l'Allemagne, Victor Margueritte, Eugène Humbert et Georges Hardy pour la France, Alexandra Kollontaï pour la Russie. L'affirmation de Wilhelm Reich selon laquelle la Ligue "réunissait les sexologues et réformateurs les

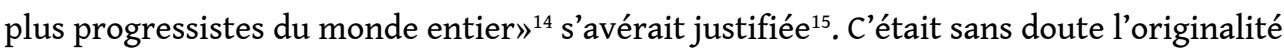
majeure de la Ligue, par rapport à certaines de ses concurrentes, comme l'INGESE (Internationale Gesellschaft für Sexualforschung) d'Albert Moll, de faire se côtoyer, au sein d'une même structure, médecins et "profanes ». Le Congrès de Londres de 1929, avec sa double direction (d'un côté le gynécologue Norman Haire, premier sexologue sur Harley Street ${ }^{16}$, de l'autre la socialiste et réformatrice Dora Russell) résumait bien cette volonté de convergence, qui n'allait pas parfois sans soulever l'irritation des médecins, soucieux des privilèges de l'expertise ${ }^{17}$.

7 Au-delà de ces querelles de prééminence, cette tension, constante, entre principes scientifiques et objectifs réformistes, constituait à la fois un atout et un handicap pour la Ligue. Une incertitude planait en effet sur la nature exacte de l'organisation et en particulier sur la manière dont elle entendait mettre la science au service de l'émancipation sexuelle. Selon le Dr Brupbacher: «Nous ne sommes pas et nous ne voulons pas être un club de discussion. Nous ne sommes pas davantage un congrès 
d'endocrinologues ou de spécialistes; nous ne sommes pas non plus simplement des sexologues, mais nous voulons aussi être des réformateurs sexuels ${ }^{18}$. Comparativement aux autres congrès ${ }^{19}$ qui se tenaient à la même époque, la Ligue consacrait d'ailleurs relativement peu de place au débat sur la sexologie en tant que telle. Le statut de la discipline même demeurait incertain. Comme le soulignait Hirschfeld, rendant hommage à Iwan Bloch, ainsi qu'à ses deux collègues Ellis et Forel, absents des Congrès, "la sexologie a grandi à la dignité de science indépendante seulement depuis le début du présent siècle ${ }^{20}$ et s'il proposait une chronologie indicative des étapes de la constitution d'une véritable science sexuelle ${ }^{21}$, il prenait soin d'inscrire l'émergence de celle-ci dans une perspective plus large: celle des mouvements féministes et abolitionnistes, de la ligue malthusienne et des groupements eugénistes, du WhK et de la société pour la réforme du mariage. La science, telle qu'elle était ici définie, ne pouvait se résumer aux différentes branches qui la constituaient, qu'il s'agisse de la biologie, de la psychologie, de la psychanalyse, de la sociologie..., même si celles-ci étaient mises à contribution. Il s'agissait bien plutôt d'une utopie scientifique qui devait servir de base à une "nouvelle» morale, rationnelle et humaniste, permettant l'épanouissement de l'individu et disqualifiant définitivement l' » ancienne » morale, dérivée de la «théologie », désignée comme le principal vecteur d'» arriération ».

8 Cette foi dans le rôle de la science, qui n'était pas dépourvue de naïveté, ni grosse de dérives possibles ${ }^{22}$, n'était évidemment pas propre à la Ligue. Elle trouvait cependant un écho particulier au sein de celle-ci du fait des engagements initiaux de Magnus Hirschfeld. Ainsi qu'il le rappelait lui-même : «Dans le combat de toute une vie contre l'ignorance et l'injustice en matières sexuelles, j'ai eu comme devise 'Per scientiam ad justiciam' [il s'agissait de la devise du WhK]. Ce but n'a pas encore été atteint. Mais il sera atteint. Le pouvoir de la vérité une fois qu'elle a été reconnue et énoncée garantit qu'il en sera ainsi. Je conclus avec l'espoir que ce Congrès fera un pas vers la réalisation de cet idéal $»^{23}$. L'utopie se parait d'accents prophétiques. Le militant néo-malthusien Eugène Humbert, absent du Congrès de Londres, adressa ainsi une lettre vibrante aux organisateurs: "Nous avons la foi la plus vive dans une régénération des peuples libérés des entraves ancestrales : l'ignorance, la superstition et la peur. Nous avons confiance que nos efforts ne resteront point stériles. Marchons résolument dans le chemin des réalisations éducatives et légales, aidons de toutes nos forces à la transformation des mœurs barbares que nous ont léguées les civilisations passées et éclairons de toute notre conscience la voie dans laquelle pourra enfin s'engager l'humanité de demain $»^{24}$. C'était l'idée d'un droit au bonheur et à la satisfaction sexuelle qui s'affirmait ici, comme portée par l'enthousiasme des premiers Congrès. Le passage des discours à la pratique allait pourtant s'avérer particulièrement malaisé. Eu égard aux dimensions de cet article, nous choisirons de présenter, comme exemplaire de ces difficultés, la question homosexuelle, un des axes majeurs du combat d'Hirschfeld au moment de la fondation de la Ligue.

\section{L'exemple de l'homosexualité : I'abandon des exigences initiales?}

9 Tous les Congrès de la Ligue mentionnent l'homosexualité, que ce soit sous la forme de comptes rendus scientifiques ou d'appels à la tolérance rédigés par des militants. Dans 
les deux cas de figure, cependant, les discussions suscitèrent davantage de sujets de tension que de concordance.

10 Le débat sur la nature de l'homosexualité révélait un premier point d'achoppement. Alors que Magnus Hirschfeld s'était fait connaître en systématisant la théorie d'Ulrichs sur le «troisième sexe ", et défendait l'hypothèse d'une homosexualité innée, nombre d'intervenants soutenaient l'idée d'une homosexualité acquise. Lors du Congrès de Berlin de 1921, le Dr Rogge revint sur les recherches de Steinach et la possibilité de "guérir » les "pseudo-homosexuels" par un traitement hormonal approprié, tandis que les Dr Schwarz et Müller-Braunschweig s'inscrivaient dans une perspective psychanalytique. Le Dr Weil, qui s'interrogeait sur le rôle des "sécrétions internes " dans « l'intersexualité », était plus proche des conceptions d'Hirschfeld. Cette polarité ne fut jamais résolue. A Vienne, en 1930, le Dr Arthur Baum concluait qu'il n'y avait pas d'homosexualité "constitutionnelle», et que celle-ci fonctionnait comme un "paravent» pour d'autres problèmes, comme le narcissisme, la frigidité ou l'impuissance, dont certains n'étaient pas forcément de nature sexuelle. A Brno, en 1932, en revanche, Hirschfeld présenta des statistiques montrant que 3\% de la population serait homosexuelle ou bisexuelle tandis que le Dr Abraham développait ses recherches sur le transsexualisme.

11 Dans tous les cas, l'enjeu restait la dépénalisation de l'homosexualité, en particulier en Allemagne où le débat autour du $\$ 175$ mobilisait les mouvements homosexuels et divisait l'opinion ${ }^{25}$. Le WhK qui se voulait apolitique, tout comme la Ligue, cherchait cependant à s'assurer du soutien des partis représentés au Reichstag : le SPD et le DDP se montrèrent conciliants, le KPD s'engageant activement dans la lutte, d'autant plus facilement que l'URSS avait aboli les lois discriminatoires. La situation était cependant loin d'être claire, la tolérance affichée par le parti communiste n'étant pas forcément partagée par l'ensemble des militants tandis que la position soviétique n'était ellemême pas dépourvue d'ambiguïté ${ }^{6}$. La Ligue se fit l'écho de ces débats. À Copenhague, Felix Halle, juriste et membre du KPD, consacra un long développement à la dépénalisation de l'homosexualité dans le cadre du projet de réforme pénale de 1927, alors que Kurt Hiller ${ }^{27}$, proche collaborateur d'Hirschfeld, renvoyant dos à dos « révolutionnaires » désinformés et législateurs « réactionnaires », s'en prit à l'écrivain communiste Henri Barbusse, qui, dans la revue parisienne Les Marges, stigmatisait l'homosexualité comme une forme de dégénérescence bourgeoise. Hiller, qui était déjà intervenu à Berlin sur "Le droit et les minorités sexuelles ", avait publié en 1922 une brochure, $\$ 175$ : la honte du siècle, où il dénonçait la pusillanimité des partis politiques vis-à-vis de la question homosexuelle. Fondateur en 1920 d'un Comité d'action (Aktionausschuss) qui échoua à unifier les mouvements homosexuels allemands, Hiller avait créé en 1925 le Cartel pour la réforme du Code pénal en matière sexuelle (Kartell für die Reforme des Sexualstrafrechts). Réunissant différents mouvements de réforme sexuelle, le Cartel inscrivait les revendications homosexuelles dans une perspective plus large, et aurait ainsi servi, selon Ralph Dose, de modèle à la Ligue. Son contreprojet de loi, en 1927, se heurta à une fin de non-recevoir, suscitant cependant un ardent débat dans la presse. L'action du Cartel et celle de la Ligue semblèrent aboutir en 1929 au moment où un nouveau projet de loi prévoyait la décriminalisation des rapports homosexuels entre adultes consentants ${ }^{28}$. Il n'entra pas en vigueur. Le WhK subit alors une crise interne, conduisant à la démission de Magnus Hirschfeld, qui choisit de se consacrer à ses activités internationales. 
12 L'important, en la matière, était, selon Hirschfeld, de rompre la « conspiration du silence ${ }^{29}$. Or, alors que le Congrès de Berlin avait consacré sept communications à l'homosexualité, celle-ci n'était plus abordée, à partir de 1929, que de manière incidente. Ce repli, s'il s'expliquait en partie par la crise de la section allemande, qui avait été la plus vocale sur le sujet, tenait aussi à des considérations internes à la Ligue. À Londres, c'est Norman Haire lui-même qui souhaita limiter les interventions sur le sujet, suite aux critiques formulées par Albert Moll ${ }^{30}$ qui aurait laissé entendre que la Ligue accordait trop d'importance aux « anomalies sexuelles »: «Bien sûr cela n'était pas vrai, et de toute façon il y a tant de choses d'un plus grand intérêt général, que nous ne pouvons pas trouver de place sur le programme du Congrès pour la discussion de l'homosexualité ou d'autres variations de la norme $»^{31}$, écrivait-il à Dora Russell. Souci de respectabilité, ambition personnelle qui le poussaient à chercher la reconnaissance du monde médical, les motivations de Haire étaient multiples, et ont été justement dénoncées par Ivan Crozier. Faut-il voir là un exemple des limites de la coopération entre médecins et militants ? On doit cependant relever qu'à la même époque la $\mathrm{BSSSP}^{32}$, dont Haire était également membre, faisait preuve d'une grande prudence visà-vis d'un sujet encore "tabou » en Angleterre. L'année 1928 avait vu l'apogée de la campagne lesbophobe orchestrée autour du livre de Radclyffe Hall, Le Puits de solitude, finalement interdit. Si l'écrivaine ne semble pas avoir souhaité intervenir lors du Congrès de Londres ${ }^{33}$, la question de l'homosexualité féminine y fut abordée à plusieurs reprises, en dépit des vœux de Haire, mais uniquement dans le cadre du débat sur la censure $^{34}$, et toujours dans une perspective intellectuelle. À Vienne en 1930, les allusions furent encore plus limitées, et recentrées sur la question de l'homosexualité «de situation». Ernst Toller et Elgar Kern abordèrent ainsi le problème de l'homosexualité en prison, tandis que le Dr Fritz Wittels faisait de l'homosexualité masculine l'une des conséquences de la «misère sexuelle » : c'était selon lui parce que les femmes allemandes faisaient preuve d'une trop grande moralité qu'elles conduisaient les hommes frustrés à se tourner vers les rapports homosexuels. L'objectif premier de la réforme pénale semblait bien être passé au second plan. L'argumentation croissante en faveur d'une homosexualité acquise, qui allait de pair avec l'évolution de la recherche médicale dans les années 30, minait de fait en partie le combat pour la dépénalisation.

13 Ce combat, en outre, ne trouvait pas forcément d'écho au sein des branches nationales. Les sections hollandaise, française et espagnole préférèrent ne pas adopter le manifeste général de la Ligue et produire une version plus adaptée, selon elles, aux réalités de leur pays ${ }^{35}$. La question homosexuelle connut ainsi un traitement différencié selon les branches. Alors que la section hollandaise, très radicale, prônait la dépénalisation de l'homosexualité entre adultes consentants et l'égalité sociale et légale pour les homosexuels, hommes et femmes, en liaison avec le NWHK, l'association de défense homosexuelle néerlandaise modelée sur le $\mathrm{WhK}^{36}$, la branche espagnole ${ }^{37}$, fondée dans le contexte favorable de la Seconde République, et inspirée par la section française, se montrait très réticente. Composée essentiellement de médecins et de juristes, elle apparaissait comme une organisation élitiste, sans doute la plus conservatrice de toutes les branches de la L.M.R.S., et dont les préoccupations, proches de celles des classes moyennes et encore marquées par le catholicisme, tranchaient avec celles des mouvements anarchistes alors très présents dans les milieux populaires espagnols. Aucun des deux courants ne témoignait par ailleurs de sympathie pour l'homosexualité, le principal enjeu des réformes étant le droit au divorce, finalement 
obtenu en $1932^{38}$. Dans le cas français, l'indifférence dominait, même si l'on doit souligner qu'à la différence des pays voisins, l'homosexualité n'y était pas condamnée, ce qui n'empêchait pas l'hostilité et le harcèlement policier. La question du contrôle des naissances dominait les débats. Selon Mathilde Chenin, la «Ligue de la Régénération humaine » ou « Pro Amor(e)», fondée en 1928, permit de donner un nouveau souffle au mouvement néo-malthusien français qui avait subi de plein fouet les persécutions consécutives à la loi de $1920^{39}$. Elle fut reprise en main, à partir de 1930, par le Comité français de la L.M.R.S dont les principaux acteurs, Berty Albrecht, Victor Basch et le Dr Sicard de Plauzole, organisèrent des conférences et la publication de la revue Le Problème sexuel de 1933 à 1935. La question homosexuelle n'y fut jamais abordée.

\section{L'échec de la Ligue : le rejet de l'option révolutionnaire?}

14 Le Congrès de Brno, qui devait originellement se tenir à Moscou, fut le dernier organisé par la Ligue ${ }^{40}$. Les meetings prévus à Paris, puis à Chicago, en 1933, n'eurent pas lieu. L'arrivée des nazis au pouvoir en Allemagne, suivie de la mise à sac de l'Institut für Sexualwissenschaft de Magnus Hirschfeld, porta un coup fatal à l'organisation, qui fut officiellement dissoute en 1935. Le but de la Ligue avait été de convaincre les gouvernements de la rationalité de la réforme sexuelle. Alors que l'Europe affrontait la dépression économique et voyait monter la menace fasciste, de telles préoccupations semblaient dépassées.

15 Dans La Révolution sexuelle, Wilhelm Reich consacre une partie de son analyse à l'échec de la Ligue. Selon lui, la « crise sexuelle » doit être comprise comme « le conflit entre la moralité qui est imposée à l'ensemble de la société par une minorité, dans l'intérêt du maintien de son pouvoir, et les besoins sexuels de l'individu »". Aussi les «libéraux, comme Magnus Hirschfeld », ou les "réformistes » en général, sont-ils voués à l'échec puisqu'ils entendent résoudre la misère sexuelle sans remettre en cause le système qui la produit. Sont particulièrement visées l'institution du mariage monogame et l'abstinence sexuelle obligatoire pour la jeunesse, qui contribuent à la reproduction de cet ordre social autoritaire, ce que la «science objective » ou «apolitique » tolère au nom de la $"$ spiritualisation des relations sexuelles $»^{42}$. Citant la déclaration publiée par Norman Haire et J.H. Leunbach après la mort de Magnus Hirschfeld, où ils expliquaient la dissolution de la Ligue du fait des "conditions économiques et politiques en Europe ", de la faillite des branches nationales française et espagnole et surtout des "divergences d'opinion» entre les membres quant au "maintien de l'apolitisme primitif de la Ligue », Reich conclut: "C'était la fin d'une organisation qui avait tenté de libérer la sexualité dans le cadre de la société réactionnaire $»^{43}$.

16 Les principaux problèmes portaient sur la définition de la réforme sexuelle et le rôle assigné aux élites dans l'élaboration d'une nouvelle morale sexuelle. En 1928, MerrittHawkes opposait ainsi les « scientifiques qui ont la connaissance des faits sexuels » et la "masse» prisonnière de ses "préjugés et tabous", tout en reconnaissant qu'il appartenait aux scientifiques d'aider « les gens ordinaires » dans leur vie quotidienne, « faisant surgir le bonheur là où il y avait le chagrin et apportant la satisfaction là où il n'y avait que le vide $»^{44}$. Pour le communiste Felix Halle, au contraire : «Une réforme sexuelle ne peut jamais être l'œuvre d'un seul, ni d'un petit groupe scientifique aussi haut placé soit-il. [...] La transformation des relations sexuelles, l'accomplissement de la 
révolution sexuelle ne peuvent être que l'œuvre de la masse nécessiteuse elle-même $»^{45}$. Pour Halle, la réforme sexuelle ne pouvait se comprendre que dans une perspective marxiste, c'est-à-dire sur la base du matérialisme scientifique. Si le KPD devait, selon lui, soutenir les efforts de la Ligue, la révolution socialiste restait le meilleur moyen d'accomplir la révolution sexuelle. Il rappelait que la révolution russe avait précipité une "réforme sexuelle radicale » en URSS, dont les acquis étaient soulignés dans la communication de G. Batkis et L. Gurwitsch consacrée à "La réforme sexuelle en URSS » et dans celle du Dr Pasche-Oserski sur "La législation pénale en matière de sexualité en URSS ». Pour ce dernier, l'URSS était appelée à servir de modèle aux autres pays, point de vue alors couramment partagé par nombre de réformateurs ou d'intellectuels. Faut-il pour autant considérer qu'il était «impossible d'atteindre les objectifs de la Ligue sans combattre simultanément pour une révolution socialiste » et affirmer, comme Leunbach, que la Ligue était "condamnée à l'impuissance parce qu'elle n'a pas rejoint le mouvement ouvrier $»^{46}$ ? Anita Grossmann souligne à juste titre que le KPD et la Ligue, malgré ses divisions entre une tendance libérale anglosaxonne ${ }^{47}$ et une tendance pro-soviétique davantage germanique et scandinave, partageaient la même foi dans le pouvoir de la science et le rôle régulateur de l'État en matière sexuelle. Si la désagrégation de l'État-providence en Allemagne à partir des années 1931-1932 et l'échec de la réforme sexuelle (notamment homosexuelle) ouvraient la voie aux demandes plus radicales d'organisations comme le Sexpol de Reich, celui-ci au final ne se trouva pas davantage en mesure de susciter des changements réels. Le durcissement de l'URSS sur les questions sexuelles, à partir de 1934-1935, devait par ailleurs porter le doute sur le potentiel émancipateur du modèle communiste en ces matières.

17 Il est difficile, dès lors, d'apprécier l'influence de la Ligue, d'autant que l'examen des conditions de sa réception est à peine engagé ${ }^{48}$. Sur cette question également, il semble que les avis aient divergé au sein de la Ligue, entre le souci d'une reconnaissance officielle, garantie possible d'un impact politique, et la volonté de rester à l'écart des pressions institutionnelles, manière de préserver une certaine radicalité de pensée. J. $\mathrm{H}$. Leunbach, en tant que secrétaire général de la Ligue, se réjouit ainsi, dans son discours de bienvenue au Congrès de Copenhague, que la cérémonie n'ait pas donné lieu à une réception officielle ou aux discours « verbeux » de représentants de l'État, de l'Église ou de toute autre instance de pouvoir ${ }^{49}$, mais se dit confiant que dans les jours qui suivent, et malgré le caractère "spartiate » de l'accueil, des questions de la plus grande importance seront abordées, suscitant de nouvelles impulsions pour les travaux à venir. De fait, et même si les membres de la Ligue furent invités, à Vienne, à rencontrer le maire de la ville à la fin du congrès ${ }^{50}$, le Congrès de Brno fut, selon le Dr Rosenthal ${ }^{51}$, le premier à revêtir un caractère "semi-officiel ", puisqu'il put se tenir dans les locaux de la faculté de médecine et qu'il fut salué à la fois par les autorités de la faculté, le régime tchèque et les villes de Brno et de Prague. Le président Masaryk, un ancien professeur de philosophie, envoya un télégramme de bienvenue au Congrès, accompagné de ses meilleurs vœux. Bien que provinciale et excentrée, et peut-être moins prestigieuse que d'autres destinations, Brno, patrie de Gregor Mendel, le fondateur de la génétique, offrait un terrain favorable à l'étude des questions de sexologie, d'autant que la législation tchèque très avancée en matière sexuelle avait été saluée à plusieurs reprises par le Congrès.

18 Une autre mesure de l'influence de la Ligue pourrait être tentée à travers les comptes rendus parus dans la presse. Ivan Crozier précise ainsi que les publications 
scientifiques, comme The Lancet ou le British Medical Journal n'avaient recensé que les communications présentant un « intérêt médical » au détriment des autres travaux. On en trouve cependant un écho dans la presse généraliste à partir du Congrès de Londres, peut-être grâce à l'action très médiatique de Norman Haire. Le Times y consacra deux articles, le premier reprenant en partie le discours d'ouverture de Magnus Hirschfeld, le second se focalisant sur la question de la censure, tandis que Le Temps, en France, s'intéressa davantage à la question du divorce, et souligna la prise de parole du délégué russe sur la situation en URSS. Dans les deux cas, il s'agissait de comptes rendus neutres, purement factuels. Le Congrès de Vienne suscita également un certain intérêt, en dépit d'une atmosphère trouble. Le Berliner Tageblatt du 17 septembre 1930 consacra un assez long article à l'ouverture du Congrès. Le journal, qui, déjà dans les années vingt, avait suivi avec sympathie le combat de Hirschfeld pour l'abolition du \$175, livrait un long passage de son intervention où il appelait entre autres au «droit à l'amour » des «minorités sexuelles». Le Times note cependant le 18 septembre 1930 que « le bâtiment du Congrès est fortement gardé du fait de précédentes manifestations d'hostilité à l'égard de la Ligue pour la Réforme Sexuelle de la part de membres du parti clérical » ce qui rappelait les attentats dont Hirschfeld, représentant alors le WhK, avait pu être l'objet lors de conférences à Munich. De manière beaucoup moins alarmante, Dora Russell et Norman Haire avaient souligné, lors de l'ouverture du Congrès de Londres, que son organisation avait été laborieuse du fait de la tradition de «pruderie sexuelle et d'hypocrisie » propre à l'Angleterre, « le pays le plus réactionnaire sur la question sexuelle $»^{52}$.

19 Les principes fondamentaux de la Ligue soulignaient que celle-ci «ne doit pas se confiner à l'étude des problèmes sexuels. Son but premier est d'obtenir des réformes pratiques pour le bien de l'humanité par l'application de la connaissance dérivée de l'étude de ces problèmes ${ }^{53}$. Dans son autobiographie, Dora Russell note, a posteriori, que le principal problème de la Ligue était que «les contributions étaient presque toutes destinées à informer ou influencer l'opinion publique plutôt qu'à organiser l'action politique [...] au total [ses] collègues érudits se contentaient de décrire l'état de la connaissance et de la pratique publique, d'exposer l'inhumanité des lois sans envisager de façon sérieuse d'y remédier $»^{54}$.

20 Organisme de coordination à vocation internationale, la Ligue n'était pas armée pour agir sur le terrain et mener une action de proximité. Une telle tâche était logiquement dévolue aux branches nationales et aux mouvements affiliés mais ceux-ci conservaient la maîtrise de leur agenda, poursuivant leurs propres objectifs. Selon Ralph Dose ${ }^{55}$, en prétendant faire la synthèse d'aspirations souvent contradictoires, la Ligue avait été incapable de satisfaire quiconque. Ni les homosexuels dont les revendications étaient noyées dans la masse, ni les féministes brimées au sein d'un mouvement majoritairement masculin, ni les néo-malthusiens pris au piège du débat sur l'eugénisme et la misère sociale. Sa faillite, pourtant, ne saurait être totale: forum international où pouvaient être discutés des sujets d'intérêt aussi bien médical, social que moral, la Ligue avait permis la diffusion des idées nouvelles dans de très nombreux pays, et constitué un espace de réflexion unique où pouvaient se confronter médecins et réformateurs sexuels, tenants d'une "révolution socialiste » et libéraux anglosaxons, humanistes et utopistes de tous bords. Elle ouvrait ainsi la voie à d'autres organisations, tel que le mouvement international pour le planning familial, qui firent 
le choix, dans un contexte plus favorable, de limiter leurs ambitions pour sans doute davantage d'efficacité ${ }^{56}$.

\section{BIBLIOGRAPHIE}

BRANDHORST Henry, 2003, « From Neo-Malthusianism to Sexual Reform : the Dutch Section of the World League for Sexual Reform ", Journal of the History of Sexuality, Vol. 12, n 1, January, p. 38-67. CHENIN Mathilde, 2003, La Ligue Mondiale pour la Réforme Sexuelle sur une Base Scientifique. À travers les archives de l'Institut International d'Histoire Sociale (I.I.S.G.) - Amsterdam, mémoire de DEA sous la direction de Francis Ronsin, Université de Bourgogne, U.F.R. Droits-Lettres.

CLEMINSON Richard, 2003, «'Science and Sympathy' or 'Sexual Subversion on a Human Basis' ? Anarchists in Spain and the World League for Sexual Reform ", Journal of the History of Sexuality, Vol. 12, n 1, January, p. 110-121.

CLEMINSON Richard, AMEZUA Efigenio, 1999, « Spain : the Political and Social Context of Sex Reform », in Eder Franz X., Hall Lesley, Hekma Gert, Sexual Cultures in Europe. National Histories, Manchester, Manchester University Press.

CROZIER Ivan, 2001, « Becoming a Sexologist : Norman Haire, the 1929 London World League for Sexual Reform Congress, and Organizing Medical Knowledge About Sex in Interwar England ", History of Science, Vol. 39, Part 3, n 125, September, p. 299-329.

--, 2003, «'All the World's a Stage'. Dora Russell, Norman Haire, and the 1929 London World League for Sexual Reform Congress ", Journal of the History of Sexuality, Vol. 12, n 1, January, p. 16-37.

DOSE Ralph, 1999, « The World League for Sexual Reform : Some Possible Approaches », in Eder Franz X., Hall Lesley, Hekma Gert, Sexual Cultures in Europe. National Histories, Manchester, Manchester University Press.

GLOCK Thomas F., 2003, « Sexual Reform, Psychoanalysis, and the Politics of Divorce in Spain in the 1920s and 1930s ", Journal of the History of Sexuality, Vol. 12, n 1, January, p. 68-97.

GREENWOOD A.W. (dir.), 1931, Proceedings of the Second International Congress for Sex Research London 1930, Londres, Oliver \& Boyd.

GROSSMANN Atina, 1995, Reforming Sex. The German Movement for Birth Control and Abortion Reform 1920-1950, Oxford, Oxford University Press.

HAIRE Norman (dir.), 1930, Sexual Reform Congress, London 8-14, IX, 1929, World League for Sexual Reform, Londres, Kegan Paul.

HALL Lesley A., 1995, « 'Desinterested enthusiasm for Sexual Misconduct': the British Society for the Study of Sex Psychology, 1913-1947 », Journal of Contemporary History, Vol. 30, n 4, October, p. 665-686.

MARCUSE Max (dir.), 1927, Verhandlungen des I. Internationalen Kongresses für Sexualforschung Berlin von 10. bis 16. Oktober 1926, Berlin et Cologne, A. Marcus et E. Weber's Verlag. 
REICH Wilhelm, 2003 [1936], La Révolution sexuelle, Paris, Christian Bourgois.

RIESE H., LEUNBACH J.H. (dir.), 1929, Sexual Reform Congress, Copenhagen 1-5, VII, 1928, World League for Sexual Reform, Proceedings of the Second Congress, Copenhague, Levin \& Munksgaard.

ROSENTHAL Dr, 1932, «Warum Sexualreform ? Bericht über den fünften Internationalen Kongress der Welt-Liga für Sexualreform 1932 », Die neue Generation, vol. 28, Heft 11/12, Nov.-Dec., p. 175-179.

RUSSELL Dora, 1975, The Tamarisk Tree. My Quest for Liberty and Love, Londres, Elek.

SINCLAIR Alison, 2003, « The World League for Sexual Reform in Spain : Founding, Infighting, and the Role of Hildegart Rodriguez ", Journal of the History of Sexuality, Vol. 12, n 1, January, p. 98-109.

STEAKLEY James D., 1997, «Per scientiam ad justiciam. Magnus Hirschfeld and the Sexual Politics of Innate Homosexuality », in Rosario Vernon A. (ed.), Science and Homosexuality, New York et Londres, Routledge.

STEINER Herbert (dir.), 1931, Sexualnot und Sexualreform ; Verhandlungen der Weltliga für Sexualreform : W.L.S.R. IV. Kongress abgehalten zu Wien vom 16. bis 23. September 1930, Vienne, Elbemühl.

TAMAGNE Florence, 2000, Histoire de l'homosexualité en Europe. Berlin, Londres, Paris, 1919-1939, Paris, Seuil.

WEEKS Jeffrey, 1979, Coming Out. Homosexual Politics in Britain from the $19^{\text {th }}$ Century to the Present, Londres, Quartet Books.

WEIL Dr A. (dir.), 1922, Sexualreform und Sexualwissenschaft, Berlin, Julius Püttmann.

\section{NOTES}

1. Magnus Hirschfeld, évoquant le combat des suffragettes dans son discours d'ouverture du $3^{\text {ème }}$ Congrès de la Ligue mondiale pour la réforme sexuelle, Londres, 1929, in Haire 1930 : xi.

2. Hirschfeld avait fondé, en 1897, le WhK (Wissenschaftlich-humanitäres Komitee : Comité Scientifique humanitaire), mouvement dont l'objectif était l'abolition du $\$ 175 \mathrm{du}$ Code pénal allemand, qui criminalisait les relations homosexuelles entre hommes, et la lutte contre les préjugés par la diffusion des connaissances scientifiques. Voir par exemple Steakley 1997, Tamagne 2000.

3. Riese, Leunbach $1929: 14$.

4. Riese, Leunbach 1929 : 14 .

5. Les actes des Congrès (Berlin, 15-20 Septembre 1921 ; Copenhague, 1-5 Juillet 1928 ; Londres, 8-14 Septembre 1929; Vienne, 16-23 Septembre 1930), à l'exception du dernier, à Brno (20-26 Septembre 1932), pour lequel ne subsistent que des comptes rendus, ont fait l'objet d'une publication dans les trois langues officielles de la Ligue (anglais, français et allemand).

6. Voir note 21 .

7. Les changements à la Constitution pouvaient être faits à la majorité simple et devaient être soumis au Comité Central exécutif au moins 4 semaines avant la date du Congrès.

8. Riese, Leunbach 1929: 9.

9. Anita Grossmann (1995: 120) souligne ainsi les convergences entre le programme de la L.M.R.S. et celui de l'EpS (Einheitsverband für proletarische Sexualreform), lié au KPD, même si celuici est " plus osé ", prônant notamment « le traitement gratuit des troubles sexuels causés par le capitalisme et la famille bourgeoise.» 
10. Ce journal ne vit jamais le jour. La branche espagnole produisit cependant deux exemplaires de Sexus, la section hollandaise Sexueele Hervorming, et la branche française Le Problème sexuel.

11. Riese, Leunbach 1929 : 23.

12. Dose $1999: 243$.

13. On compte 182 adhérents individuels, dont un tiers pour la branche britannique. Lors des votes, chaque membre individuel avait une voix; les organisations pouvaient prétendre au maximum à 5 voix (1 voix pour 500 membres). Lors de l'Assemblée Générale, chaque pays était représenté par un vote.

14. Reich $2003: 103$.

15. Néanmoins Ivan Crozier remarque que le Congrès de Londres ne présentait qu'un panel restreint de scientifiques et de médecins; les psychanalystes de renom, en particulier, étaient absents.

16. Haire, «sexologue australien, homosexuel, juif, qui ne buvait pas d'alcool et souffrait de surpoids » pouvait cependant être considéré comme un "outsider» au sein du monde médical britannique très conservateur. Crozier 2001.

17. Norman Haire se plaça ainsi, lors du Congrès de Londres, en situation de concurrence face à Marie Stopes, Stella Browne et Naomi Mitchison sur la question du contrôle des naissances, dévalorisant leurs interventions comme non professionnelles. Crozier 2001.

18. Riese, Leunbach 1929 : 107.

19. Marcuse 1927, Greenwood 1931.

20. Haire 1930 : xii.

21. Il cite notamment la publication du Zeitschrift für Sexualwissenschaft (1908), la première conférence internationale sur le contrôle des naissances qui se tint à Dresde en 1911 sous l'impulsion d'Helene Stöcker et du Bund für Mutterschutz und Sexualreform, la création de l'Institut für Sexualwissenschaft à Berlin en 1919 et le premier Congrès International pour la Réforme Sexuelle sur une Base Scientifique qui se tint à Berlin en 1921, et fit ainsi figure de précurseur de la L.M.R.S. Riese, Leunbach $1929: 29$.

22. Il n'est pas possible ici de consacrer une longue analyse à la question de l'eugénisme, vis-à-vis de laquelle, cependant, des membres de la Ligue ont manifesté leur inquiétude. Lors du Congrès de Londres, le Dr Eder intervint sur le thème de « la stérilisation des inaptes ", pour la dénoncer comme incapable de résoudre le problème de l'arriération mentale et susceptible au contraire de réduire la proportion "d'individus talentueux ou géniaux ", tandis que Magnus Hirschfeld, s'il décourageait les "intersexuels» de chercher à procréer, s'opposait à l'idée de stérilisation forcée. Mathilde Chenin (2003 : 50-52) montre néanmoins que d'autres, comme la britannique Cora Hodson, ou le français Eugène Humbert, ont au contraire voulu voir dans la science eugénique un moyen de lutter contre la dégénérescence et/ou la misère sociale.

23. Haire $1930: \mathrm{xv}$.

24. Haire $1930: \mathrm{xxv}$.

25. La réforme du $\$ 175$ fut l'objet de plusieurs projets de loi, en 1919, 1925, 1927, 1933 et de multiples débats avant que le régime nazi, en 1935, ne le modifiât finalement dans un sens encore plus défavorable, marquant l'échec définitif du combat pour la dépénalisation.

26. En 1917, les lois sur la sodomie avaient été abolies, comme l'ensemble du code pénal tsariste, laissant croire à une tolérance particulière de la part du régime bolchevique. À partir de 1934, cependant, l'homosexualité, "perversion fasciste » fut à nouveau passible d'une peine pouvant s'élever jusqu'à 5 ans de travaux forcés. Il semble d'ailleurs que la répression existait déjà avant cette date et ne fit ensuite que s'accentuer. La position marxiste vis-à-vis de l'homosexualité était du reste confuse. Dans L'Origine de la famille, de la propriété privée et de l'État de Friedrich Engels, l'homosexualité était présentée comme un « vice choquant et contre-nature ».

27. Hiller, membre du groupe des Pacifistes révolutionnaires était antibolchevique. Hirschfeld était membre du SPD. 
28. Dose 1999, Tamagne 2000.

29. Haire 1930 : XIII.

30. Pour une comparaison avec les Congrès de l'INGESE qui se tiennent à Berlin en 1926 et à Londres en 1930 et se veulent uniquement scientifiques, voir Marcuse 1927, Greenwood 1931, Dose 1999.

31. Lettre du 21 février 1929 in Crozier 2001 : 316.

32. Weeks 1979, Hall 1995, Tamagne 2000. Fondée en 1914 sous le patronage de Magnus Hirschfeld, Havelock Ellis et Edward Carpenter qui en devint le président d'honneur, la BSSSP, très marquée par l'influence fabienne, faisait de la réforme sexuelle, en particulier de la dépénalisation de l'homosexualité, son objectif majeur. Néanmoins, elle ne cherchait pas être identifiée comme un mouvement homosexuel et privilégia toujours le débat à l'activisme.

33. Elle envoie un télégramme d'excuse. Norman Haire et Laurence Housman avaient, entre autres, accepté de témoigner en sa faveur lors du procès.

34. William J. Robinson évoqua la question du lesbianisme dans le cadre de la prostitution. Sur lesbianisme et censure, voir les interventions de H.F. Rubinstein, Laurence Housman, Georges Ives, Bertrand Russell in Haire 1930.

35. Ralph Dose remarque qu'il s'agit de branches fondées tardivement (1932 pour les branches hollandaise et espagnole) et de manière relativement indépendante. Cependant, la branche anglaise, sous l'impulsion de Norman Haire, choisit également de livrer une version révisée du programme de la Ligue, « moins susceptible d'offenser le public anglais » (Crozier 2001 : 312.).

36. Brandhorst $2003: 47$.

37. Cleminson, Amezua 1999 ; Cleminson 2003 ; Glock 2003 ; Sinclair 2003.

38. L'homosexualité fut également décriminalisée en 1932, sauf dans les forces armées.

39. Les Français étaient cependant peu représentés au Congrès de Londres, même si l'on doit souligner la présence de Madeleine Pelletier qui intervint à deux reprises, d'abord sur l'avortement, puis sur « Le sexe et la morale».

40. La section espagnole parvint cependant à organiser d'avril à mai 1933 une conférence consacrée aux questions de génétique et d'eugénisme mais aussi à la prostitution et à la puériculture, à laquelle assistèrent le ministre de l'Instruction publique et le Président de la République (Cleminson, Amezua 1999: 188). La section britannique survécut à travers trois associations : la Sex Education Society, la Federation of Progressive Societies and Individuals qui devint la Progressive League et la British Sexology Society, héritière de la BSSSP (Hose 1999 : 252).

41. Reich $2003: 72$.

42. Reich $2003: 86$.

43. Reich $2003: 105$.

44. Riese, Leunbach $1929: 123$.

45. Riese, Leunbach $1929: 111$.

46. Reich 2003 : 105.

47. Le choix de Moscou comme lieu du futur Congrès souleva en 1929 des objections de la part de certains participants, inquiets de l'accueil des membres non communistes en URSS. Dora Russell craignant pour sa part qu'aucun Congrès ne puisse se tenir ensuite aux Etats-Unis, souligna que l'URSS, en avance en matière sexuelle, n'avait pas besoin d'un tel rassemblement.

48. La L.M.R.S., malgré un regain d'intérêt dans les années 60 , n'a fait l'objet jusqu'à présent que de travaux dispersés. Si l'on excepte la tentative de synthèse de Ralph Dose, la plupart des auteurs se sont concentrés sur une section nationale, sans qu'il soit possible de tenter une appréciation globale de la réception critique des travaux de la Ligue (Dose 1999).

49. Il est remarquable que ce passage ait été supprimé de la traduction anglaise.

50. Chenin : 60 .

51. Rosenthal $1932: 175$.

52. Haire 1930 : xvi, xx. 
53. Haire 1930 : 593.

54. Russell 1975: 218. Haire rompra avec Dora Russell en raison de ce qu'il appelle sa « subordination de la réforme sexuelle à la politique ». Crozier $2003: 36$

55. Dose 1999 : 246.

56. Dose 1999 : 252.

\section{RÉSUMÉS}

Fondée en 1928 par Magnus Hirschfeld, la Ligue Mondiale pour la Réforme Sexuelle entendait rassembler médecins et « profanes » dans le but de diffuser dans l'opinion publique les acquis de la nouvelle "science sexuelle » et d'influencer les gouvernements dans un sens progressiste, sur des questions aussi variées que le contrôle des naissances, le mariage et le divorce, l'homosexualité, la prostitution ou l'eugénisme. Très vite pourtant, elle fut déchirée entre des tendances contradictoires, et dans l'incapacité de mener à bien ses objectifs, alors que la crise des années trente rendait son combat pour une nouvelle morale sexuelle rationnelle et humaniste de plus en plus utopique et que son pouvoir d'action se trouvait limité à la tenue de Congrès et à la dénonciation des lois injustes.

The World League for Sexual Reform was created in 1928 by Magnus Hirschfeld in order to bring about "a new attitude towards all sexual questions, based on the findings of sexual science". Gathering together doctors and lay persons, it aimed to influence governments on controversial issues such as birth control, marriage, divorce, homosexuality, prostitution or eugenics but was rapidly torn up between contradictory trends and unable to achieve its goals. At a time of economic, social and international crises, the League's appeal to a new rational and humanist sexual moral seemed more and more utopian, even more so with its action limited to the holding of Congresses and to the denunciation of unfair laws through uncommitted speeches.

\section{INDEX}

Mots-clés : homosexualité, Hirschfeld Magnus, médecine, science, sexologie, sexualité, utopie Index géographique : néo-Malthusianisme

\section{AUTEUR}

\section{FLORENCE TAMAGNE}

Florence TAMAGNE est maîtresse de conférences à l'Université de Lille 3. Historienne du genre et des sexualités, elle a publié Histoire de l'homosexualité en Europe. Berlin, Londres, Paris, 1919-1939, Paris, Seuil, 2000 et Mauvais genre? Une histoire des représentations de l'homosexualité, Paris, EDLM, 2001. 